\title{
Registro Nacional de Quemados Chile: Presentación de una plataforma online y mirada al pronóstico de pacientes no trasladados a Centro de Quemados
}

\author{
Roberto Ariel Macchiavello M. ${ }^{1}$, María Angélica Paulos P. ${ }^{1}$, Carolina Soto D.1, \\ Manuel Calcagno L. ${ }^{1}$, Carlos Barril M. ${ }^{1}$ y Cristián Arriagada I. ${ }^{1}$
}

'Hospital de Urgencia Asistencia Pública, Santiago,

Recibido 2020-11-15 aceptado 2021-02-08

Correspondencia a: Dr. Roberto Ariel Macchiavello $\mathrm{M}$

robertomac89@gmail.com
Presentation of an online National Registration Platform for severely burned patients in the Chilean Public Health System and a look into the prognosis of patients not transferred to the National Reference Burn Center

Introduction: In 2017, an online notification register, the National Burn Registry, was incorporated into the referral flow of burned patients in Chile. Aim: Through the information obtained from this platform, we describe the epidemiology of burns in Chile, and identify variables that could explain failed transfers to our burn unit. Materials and Method: Cases uploaded to this platform between July 2017 - July 2018 were analyzed. We characterize the global population and relevant variables were compared between the group of patients that failed to be transferred to the burn unit and the ones who were successfully transferred. Results: 319 patients were analyzed, 66\% men, average age 51 years, BMI of 27 and $47 \%$ with previous illnesses. Fire was the main cause of burn injury. Smoke inhalation injury was observed for $31 \%$. 107 patients failed to reach to our burn center. Transferred patients rated higher in comorbidity, severity index, total burned body surface and surgical debridement at base hospital. The group of not transferred patients rated higher in inhalation injury. Overall mortality was $20.4 \%$. Mortality was higher in non-transferred patients $(33.6 \%$ versus $13.7 \%$; $<0.001)$. Conclusions: Aside from facilitating the flow of burned patients and resources saving, a noble use of this platform has been to serve as a source of epidemiological information and implementation of public policies, which can be taken as an example by other developing countries. Also, being transferred is a protective factor for death from burn injuries. Keywords: burns, burn unit; patient transfer; public policy.

\section{Resumen}

Introducción: En el año 2017 se incorporó un registro de notificación en línea (Registro Nacional de Quemados) al flujo de derivación de pacientes quemados en Chile. Objetivo: A partir de la información obtenida de esta plataforma, se describe la epidemiología de las quemaduras y las variables que podrían explicar los traslados fallidos a nuestra unidad de quemados. Materiales y Método: Se analizaron los casos subidos a esta plataforma entre julio de 2017 y julio de 2018. Se caracterizó la población global y comparó variables relevantes entre el grupo de pacientes no trasladados a nuestra unidad y los que fueron trasladados con éxito. Resultados: Se analizaron 319 pacientes, 66\% hombres, edad promedio 51 años, IMC de $27 \%$ y $47 \%$ con enfermedades previas. El fuego fue la principal causa de quemaduras. Se observó un $31 \%$ de injuria inhaladora. 107 pacientes no se trasladaron a nuestro centro de quemados. Los pacientes trasladados puntuaron más alto en comorbilidad, índice de gravedad, superficie corporal total quemada y aseo quirúrgico en el hospital base. El grupo de pacientes no trasladados puntuó más alto en injuria inhalatoria. La mortalidad global fue 20,4\%. La mortalidad fue mayor en pacientes no trasladados $(33,6 \%$ versus $13,7 \%$; $\mathrm{p}<0,001)$. Conclusiones: Además de facilitar el flujo de pacientes y ahorrar recursos, un uso noble de esta plataforma es ser fuente de información epidemiológica y de implementación de políticas públicas, lo cual puede ser tomado como ejemplo por otros países en vías de desarrollo. Además, se demuestra que ser trasladado constituye un factor protector de muerte por quemaduras.

Palabras clave: quemados; unidad de quemados; traslado de pacientes; políticas públicas. 


\section{Introducción}

Las quemaduras son un problema de salud pública, registrándose el año 2007 por el Ministerio de Salud 6.435 egresos hospitalarios por quemaduras y 569 muertes por esta causa según el Instituto Nacional de Estadística ${ }^{1,2}$. En el año 2007 se incorporó al GES el manejo de pacientes con quemaduras graves y se designó al Hospital Urgencia Asistencia Pública como Centro de Referencia para los adultos grandes quemados, definidos por los criterios de derivación descritos en las Guías Clínicas GES ${ }^{1}$. Nuestro centro, con 10 camas de UCI y 12 camas de atención de complejidad media, atiende aproximadamente 200 pacientes con quemaduras graves por causas no laborales al año ${ }^{2}$.

Las características geográficas de Chile representan un gran desafío en la derivación y traslado de pacientes. Algunos de los problemas con el traslado se relacionan con el diagnóstico (una sobreestimación de la superficie corporal quemada y el subsiguiente "fluid creep syndrome"), la correspondencia con los criterios de derivación, el tiempo entre el evento y la notificación de derivación, y el tipo de movilización para un correcto traslado. Por otro lado, nuestras limitadas camas de UCI para la población asignada nos obligan a ser extremadamente eficientes en la aceptación o rechazo de pacientes.

Con el objetivo de mejorar estos aspectos de la derivación, en el año 2017 incorporamos un registro de notificación en línea, el Registro Nacional de Quemados ${ }^{3}$. Esta plataforma reemplazó el antiguo sistema de referencia manual de protocolos burocráticos y poco claros.

Consiste en un portal web en donde los profesionales de la salud de los departamentos de emergencia de los hospitales regionales pueden acceder a través de credenciales de usuario únicas y presentar una solicitud de traslado para los pacientes quemados que cumplen con los criterios de GES.

A su vez, los médicos del centro de referencia contarán con un perfil diferente en el portal que permite evaluar los casos subidos a la plataforma y tomar decisiones respecto a ellos (Figura 1).

El perfil del médico de urgencias de un hospital regional mostrará en su pantalla inicial los elementos "Ayuda", "Registro histórico" y "Registro de paciente". Para registrar a un nuevo paciente,

\section{RNQ | QUEMADos}

Histórico Ayuda

Histórico de Quemados

\begin{tabular}{|c|c|c|c|c|c|c|c|c|c|c|c|c|c|c|c|c|}
\hline \multirow{2}{*}{\multicolumn{2}{|c|}{$\begin{array}{l}\text { Cà Fotos } \triangle \text { Consultar } \\
\text { Código Quemado }\end{array}$}} & \multirow{2}{*}{\multicolumn{2}{|c|}{ Establecimiento }} & \multicolumn{2}{|c|}{$\checkmark$ Cambiar Estado } & Câ Bitacora & \multirow{2}{*}{$\begin{array}{l}\text { Establecimiento Destino } \\
\text { Evolución Clinica }\end{array}$} & \multicolumn{2}{|c|}{ Monitoreo Paciente } & Registro Teleasistencia & \multicolumn{3}{|c|}{ S Salir } & \multirow{2}{*}{\multicolumn{2}{|c|}{ Limpiar }} & \\
\hline & & & & Origen & Región & 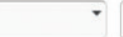 & & \multicolumn{2}{|c|}{ Indice de Gravedad } & \multirow[t]{2}{*}{ Estatus } & \multirow[t]{2}{*}{ r } & Categoría de Gravedad & • & & & \\
\hline Foto & Quemado Rut Pac & iente & Fecha Qu & emadur: & Fecha Ingreso & Mecanismo & Establecimiento & & Aceptado & & & Médico & Edad & Gravedad & Días & \\
\hline$\checkmark$ & 1086 & & $30 / 04 / 20$ & & $30 / 04 / 2020$ & Fuego & Hospital San Martín (Quillot & & No se ha asigna & do cama** & & & 31 & 150.0 & 0 & $\square$ \\
\hline$\checkmark$ & 1085 & & $27 / 04 / 20$ & & $27 / 04 / 2020$ & Agua & $\begin{array}{l}\text { Hospital Dr. Félix Bulnes Cerc } \\
\text { (Santiago, Quinta Normal) }\end{array}$ & & No se ha asigna & do cama** & & & 40 & 62.0 & 1 & \\
\hline$\checkmark$ & 1084 & & $28 / 04 / 20$ & & $28 / 04 / 2020$ & Fuego & $\begin{array}{l}\text { Hospital Presidente Carlos It } \\
\text { del Campo (Linares) }\end{array}$ & ṕñez & No se ha asigna & do cama & & & 62 & 98.0 & 2 & \\
\hline$\checkmark$ & 1083 & & $28 / 04 / 20$ & & $28 / 04 / 2020$ & $\begin{array}{l}\text { Explosión } \\
\text { gas }\end{array}$ & Hospital Base San José de O & sorno & No se ha asigna & do cama & & & 20 & 100.0 & 2 & \\
\hline$\checkmark$ & 1082 & & $28 / 04 / 20$ & & $28 / 04 / 2020$ & $\begin{array}{l}\text { Explosión } \\
\text { gas }\end{array}$ & Hospital Base San José de O & sorno & $\begin{array}{r}\text { Hospital de U } \\
\text { Pública Dr. Alej }\end{array}$ & $\begin{array}{l}\text { rgencia Asistencia } \\
\text { andro del Río }\end{array}$ & & & 23 & 106.0 & 1 & س \\
\hline$\checkmark$ & 1081 & & $24 / 04 / 20$ & & $24 / 04 / 2020$ & $\begin{array}{l}\text { Explosión } \\
\text { gas }\end{array}$ & $\begin{array}{l}\text { Complejo Hospitalario Dr. Só } \\
\text { del Río (Santiago, Puente Alt }\end{array}$ & & No se ha asigna & do cama & & & 92 & 114.5 & 2 & \\
\hline$\checkmark$ & 1080 & & $24 / 04 / 20$ & & $24 / 04 / 2020$ & $\begin{array}{l}\text { Explosión } \\
\text { gas }\end{array}$ & $\begin{array}{l}\text { Complejo Hospitalario Dr. So } \\
\text { del Río (Santiago, Puente Alt }\end{array}$ & & $\begin{array}{r}\text { Hospital de U } \\
\text { Pública Dr. Alej }\end{array}$ & $\begin{array}{l}\text { rgencia Asistencia } \\
\text { andro del Río }\end{array}$ & & & 68 & 87.0 & 2 & $\square$ \\
\hline
\end{tabular}

Figura 1. Pantalla de inicio del Registro Nacional de Quemados. Se muestra una lista de pacientes quemados cargada en la plataforma con los siguientes datos de izquierda a derecha: estado de la foto, número de solicitud, identificación del paciente, fecha de quemadura, fecha de admisión, mecanismo, hospital, estado de aceptación, contacto y nombre del médico de urgencias, edad, índice de Garcés y días de espera. Se han borrado las identificaciones de pacientes y médicos. 
debe hacer clic en el botón de registro y proceder a completar un formulario con los datos del paciente, que incluye:

1. Información básica del paciente: nombre, edad, sexo, dirección, teléfono, rut, peso, talla y enfermedades previas.

2. Detalles de la quemadura: fecha y hora de la quemadura, fecha y hora de ingreso hospitalario, quemadura en un espacio cerrado (sí/no), trauma encefalocraneal (sí/no), injuria inhaladora (sí/ no), quemadura a lo "bonzo" (sí/no), compromiso de conciencia (sí/no), politraumatismo (sí/ no), mecanismo de la quemadura y contacto del médico.

3. Diagnóstico de la quemadura: Extensión y profundidad de la quemadura por áreas anatómicas, que permite el cálculo del índice de severidad, la superficie corporal total quemada y el pronóstico.

4. Reanimación y constantes vitales: tipo de fluido de reanimación y volumen utilizado, signos vitales.

5. Tratamiento: Presencia de aseo quirúrgico, escarotomía, escarectomía, cultivos y fasciotomía, antibióticos, analgesia y fármacos vasoactivos.

6. Exámenes de laboratorio realizados.

7. Dispositivos: presencia de catéteres venosos, catéter urinario y vía arterial.

8. Fotos de las quemaduras.

En el caso del perfil de médico del centro de referencia, mostrará en su pantalla inicial los ítems "Ayuda" y "Registro histórico". Al hacer clic en este último, se mostrará una lista con todos los pacientes que se han agregado para un eventual traslado desde los hospitales regionales, como se describe anteriormente.

El médico tendrá la opción de revisar cada caso y aceptarlo, si cumple con los criterios de derivación, rechazarlo si no, o solicitar más información o fotos si es necesario. Una vez aceptado, el encargado de camas del centro de referencia podrá asignar una cama a través de la misma plataforma.

El objetivo de este trabajo es compartir nuestra experiencia con la utilización del Registro Nacional de Quemados, y a través de los datos que esta plataforma nos entrega, describir la epidemiología del Gran Quemado en Chile e identificar las variables que puedan explicar el traslado fallido de algunos pacientes a nuestro centro de referencia. Para esto último, se determinará el pronóstico y mortalidad de los pacientes no trasladados comparándola al grupo de pacientes que lograron trasladarse con éxito a nuestra unidad.

\section{Materiales y Método}

Este estudio fue diseñado utilizando la metodología de cohorte histórica. Analizamos todos los casos subidos a la plataforma del Registro Nacional de Quemados en el período de un año, entre julio de 2017 y julio de 2018, alcanzando un total de 319 pacientes, sin casos perdidos. Este universo no incluyó a los pacientes menores de 15 años, ya que estos pacientes están excluidos del Registro Nacional de Quemados por ser tratados en las unidades de quemados de los hospitales pediátricos. Todos los datos fueron debidamente solicitados y autorizados por las autoridades correspondientes del Ministerio de Salud de Chile.

Se comenzó con la caracterización de la población global de pacientes quemados provenientes de la plataforma, y luego se definieron los dos grupos a analizar: un grupo de pacientes que fueron trasladados exitosamente a la unidad de quemados y el grupo de pacientes que no pudieron ser trasladados a la unidad de quemados.

Se analizaron las variables relevantes del grupo no trasladado y se compararon con las del grupo trasladado. Nuestra principal variable de exposición es haber sido trasladado y la variable de resultado es haber fallecido. Los datos sobre los factores de riesgo de muerte (variable de confusión) se recopilaron de acuerdo con factores de riesgo conocidos sobre quemaduras identificados en la literatura, incluyendo edad, sexo, superficie corporal total, grado de quemadura, injuria inhalatoria, comorbilidad, índice de masa corporal (IMC) e índice de severidad. La edad fue una variable dicotómica mayor o menor de 65 años, y el IMC se categorizó como obeso, con sobrepeso y normal. El índice de pronóstico de Garcés se clasificó en: 21-40 puntos - leve, 41-70 puntos moderado, 71-100 puntos - grave, 101-150 puntos - crítico y $>150$ puntos - supervivencia excepcional. Las regiones de Chile se agruparon en tres áreas: Norte (Regiones I, II, III, IV, XV), Central (Regiones V, VI, VII, XIII, XVI) y Sur (Regiones VIII, IX, X, XI, XII, XIV). Se buscaron modificadores de efectos sin encontrarlos. El tamaño de muestra estimado para la comparación de las dos muestras fue de 104 pacientes para cada grupo.

\section{Análisis estadístico}

En el presente estudio, las diferencias entre variables categóricas se analizaron con $\mathrm{Chi}^{2}$ o prueba de Fisher, según corresponda. La edad y la superficie corporal quemada se analizaron con prueba no paramétrica de Mann-Whitney. Los cálculos de supervivencia con curva de Kaplan Meier y regresión de 
Cox multivariante, y el cálculo de LD50 con 50\% de sensibilidad en modelo univariado con el evento de morir y superficie corporal quemada. Un valor de $\mathrm{p}$ de menos de 0,05 se consideró estadísticamente significativo. Los análisis estadísticos se realizaron con el programa Stata ${ }^{\circledR}$ versión 11.2 (Stata Corporation, College Station, Texas).

\section{Resultados}

319 pacientes fueron ingresados al Registro $\mathrm{Na}-$ cional de Quemados entre julio de 2017 y julio de 2018 desde hospitales en todo el país. 107 (34\%) pacientes no llegaron al Centro de Referencia.

En cuanto a las características de la población general, el $66 \%$ eran hombres $(n=213)$ y el $33 \%$ mujeres $(n=106)$, una edad promedio de 51 años (DE 20,7; rango de 15 a 94 años) y el 28,5\% eran mayores de 65 años $(n=91)$. Un IMC medio de 27 (DE 5,4; rango de 17,8 a 66,4 ) con el $69 \%$ de los pacientes con sobrepeso (IMC > 25) y el $20 \%$ de los pacientes obesos (IMC > 30). 47\% de los pacientes tenían enfermedades previas $(\mathrm{n}=149), 56 \%$ mujeres y $42 \%$ hombres, con $13,8 \%$ de pacientes hipertensos, $11 \%$ de consumo de alcohol, $8,5 \%$ de pacientes diabéticos, 4,4\% de fumadores, 3,8\% de pacientes con enfermedad psiquiátrica, el $3 \%$ de los pacientes epilépticos, el 2,5\% de los pacientes con EPOC y el $1,3 \%$ de los pacientes con hipotiroidismo. La injuria inhalatoria estaba presente en el 31\% de los casos, pero el politraumatismo y el traumatismo encefalocraneal fueron poco frecuentes, con un $4,1 \% \mathrm{y}$ un $2,2 \%$, respectivamente (Tabla 1 ). En cuanto a la estacionalidad, la demanda aumenta en invierno, con un $24 \%$ de las solicitudes realizadas en junio y julio. El momento del día en el que los pacientes sufren quemaduras con mayor frecuencia es a las 14:00 horas (DE 6 horas). La tasa de quemaduras más alta considerando población por región corresponde a Arica y Parinacota en el Norte de Chile, con una tasa de 7,52, en comparación con la tasa de la Región Metropolitana de 1,94. En cuanto a las distancias estimadas entre el hospital base y el centro de referencia, el 39\% de los pacientes $(n=125)$ se encontraban a más de $250 \mathrm{~km}$ del centro de referencia.

En cuanto a la caracterización de las quemaduras, en el $61 \%$ el mecanismo es fuego $(\mathrm{n}=197,8,8 \%$ autoinfligido), $17,5 \%$ escaldadura $(n=56), 10,97 \%$ explosión de gas $(n=35), 5,96 \%$ eléctrico $(n=19)$ y $3,76 \%$ químico $(n=12)$ (Tabla 2$)$.

La profundidad más frecuente de la lesión por quemadura son las quemaduras de espesor parcial y las quemaduras de espesor total en una presentación mixta (Tabla 3).

La mediana del índice de severidad de Garcés fue de 93 (rango intercuartílico 66-130) y las categorías de pronóstico se muestran en la Tabla 4.

Al comparar ambos grupos (pacientes trasladados y no trasladados), no se encontraron diferencias estadísticamente significativas en cuanto a sexo, edad, porcentaje de adultos mayores, IMC, agente y distribución geográfica de los pacientes quemados.

Los pacientes trasladados puntuaron más alto en comorbilidad, índice de gravedad (Figura 2), quemaduras graves o críticas, superficie corporal total quemada y quemaduras mixtas con presencia de quemaduras de espesor total. A su vez, el grupo de pacientes no trasladados puntuaron más alto en presencia de injuria inhalatoria, mal pronóstico con

Tabla 1. Características demográficas y comorbilidades de la población, por estatus de traslado al centro de quemados

\begin{tabular}{|c|c|c|c|}
\hline Características demográficas & $\begin{array}{c}\text { Pacientes trasladados } \\
\text { (212) }\end{array}$ & $\begin{array}{l}\text { Pacientes no trasladados } \\
\text { (107) }\end{array}$ & $\begin{array}{c}\text { Significancia estadística } \\
\text { (valor de } p \text { ) }\end{array}$ \\
\hline Sexo (hombre/mujer) & $137: 75$ & $76: 31$ & NS* \\
\hline Mediana de edad (p25-p75) & $51(34-69)$ & $50(33-65)$ & $\mathrm{NS} * * *$ \\
\hline Adulto mayor (> 65 años) & $29,9 \%$ & $25,2 \%$ & NS* \\
\hline Norte/Centro/Sur de Chile & $29: 131: 52$ & $15: 75: 17$ & $\mathrm{NS} * * *$ \\
\hline TEC & $1,9 \%$ & $1,9 \%$ & $\mathrm{NS} * * *$ \\
\hline Injuria inhalatoria & $25,0 \%$ & $42,0 \%$ & $\mathrm{p}=0,003^{*}$ \\
\hline Politrauma & $4,7 \%$ & $2,8 \%$ & $\mathrm{NS} * * *$ \\
\hline
\end{tabular}

Test estadístico utilizado: Test chi cuadrado de Pearson*, Test exacto de Fisher** y Test de Mann Whitney***. 
Tabla 2. Agente causal por estatus de traslado al centro de quemados

\begin{tabular}{|c|c|c|c|}
\hline Agente causal & $\begin{array}{l}\text { Pacientes trasladados } \\
\text { (212) }\end{array}$ & $\begin{array}{c}\text { Pacientes no trasladados } \\
\text { (107) }\end{array}$ & $\begin{array}{c}\text { Significancia estadística } \\
\text { (valor de p) }\end{array}$ \\
\hline Escaldadura & $16,1 \%$ & $20,6 \%$ & NS* \\
\hline Explosión de gas & $9,0 \%$ & $15,0 \%$ & NS* \\
\hline Fuego & $64,0 \%$ & $57,0 \%$ & NS* \\
\hline
\end{tabular}

Test estadístico utilizado: Test chi-cuadrado de Pearson* y Test exacto de Fisher**.

Tabla 3. Características de las quemaduras de la población por estatus de traslado al centro de quemados

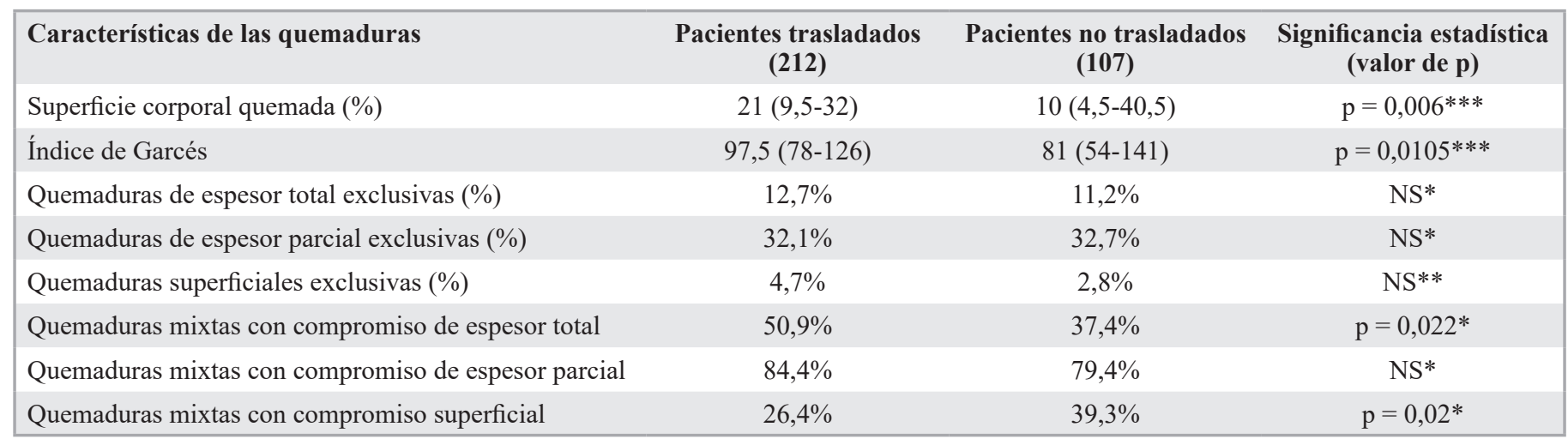

Test estadístico utilizado: Test chi-cuadrado de Pearson*, Test exacto de Fisher** y Test de Mann Whitney ***.

Tabla 4. Pronóstico de la quemadura por estatus de traslado a centro de quemados

\begin{tabular}{|cccc|}
\hline Pronóstico & $\begin{array}{c}\text { Pacientes trasladados } \\
(\mathbf{2 1 2})\end{array}$ & $\begin{array}{c}\text { Pacientes no trasladados } \\
(\mathbf{1 0 7 )}\end{array}$ & $\begin{array}{c}\text { Significancia estadística } \\
(\mathbf{v a l o r} \text { de p) }\end{array}$ \\
Leve & $3,3 \%$ & $14,0 \%$ & $\mathrm{p}<0,005^{* *}$ \\
Moderado & $14,2 \%$ & $30,8 \%$ & $\mathrm{p}<0,005^{*}$ \\
Grave & $36,0 \%$ & $20,6 \%$ & $\mathrm{p}<0,005^{*}$ \\
Crítico & $32,2 \%$ & $11,2 \%$ & $\mathrm{p}<0,005^{*}$ \\
Sobrevida excepcional & $14,2 \%$ & $23,4 \%$ & $\mathrm{p}=0,02^{*}$ \\
\hline
\end{tabular}

Test estadístico utilizado: Test chi-cuadrado de Pearson* y Test exacto de Fisher**.

sobreviva excepcional y quemaduras mixtas con presencia de quemadura superficial.

Los pacientes trasladados al centro de quemados se habían sometido a un aseo quirúrgico en el hospital base con más frecuencia (54\%) que los pacientes que no fueron trasladados $(33 \%)$. La letalidad global fue del $20,4 \%$ (13\% en pacientes trasladados y $33,6 \%$ en pacientes no trasladados). La letalidad fue mayor en los pacientes no trasladados que en los trasladados $(33,6 \%$ versus $13,7 \%$; $p<0,001)$.

Ser trasladados, por tanto, es un factor protector para morir por quemaduras con un Hazard Ratio 0,17 (95\% CI 0,09-0,30), ajustado por sexo, edad, comorbilidad, superficie de la quemadura, quema- 


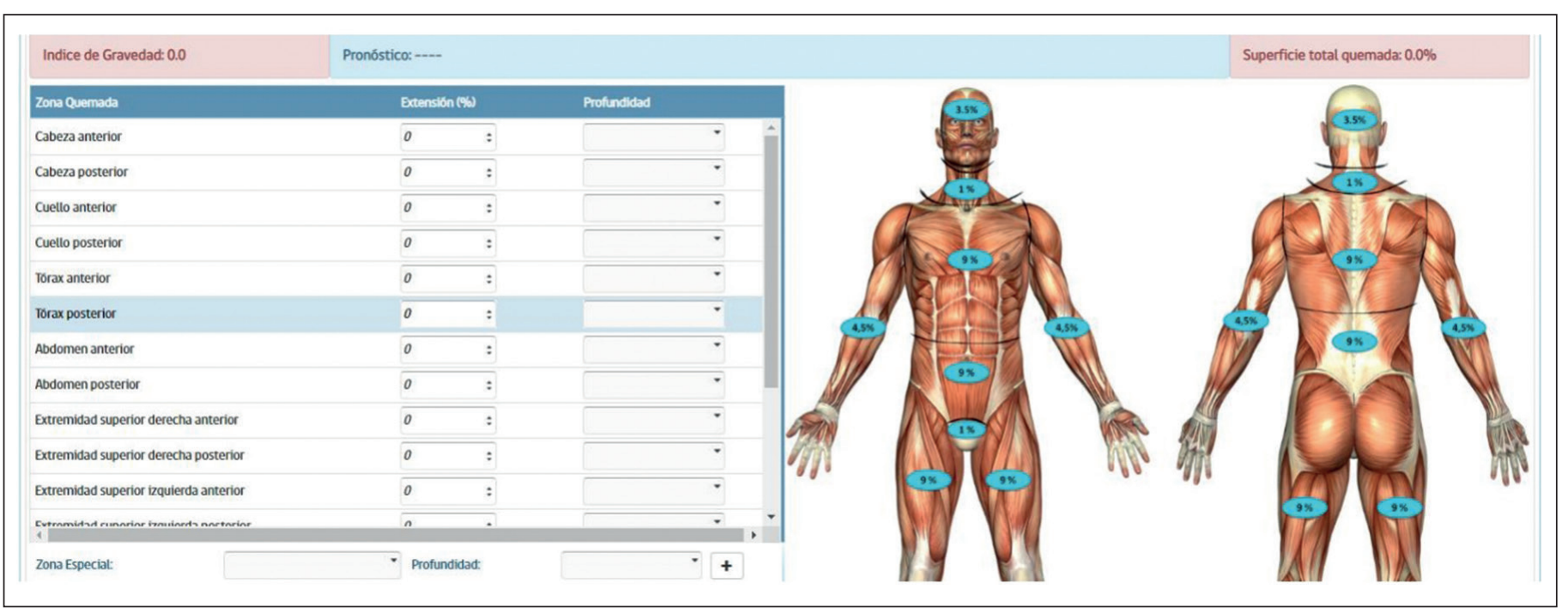

Figura 2. Hoja de registro de la superficie corporal del Registro Nacional de Quemados. El médico de urgencias puede completar la ubicación, profundidad y superficie corporal total de la quemadura.

Tabla 5. Probabilidad de muerte de los pacientes por diferentes variables, incluido el estatus de traslado

\begin{tabular}{|lccc|}
\hline Variable & Hazard Ratio & IC 95\% & Valor de p \\
Trasladado/No trasladado & 0,31 & $0,19-0,51$ & $<0,001$ \\
Sexo (hombre/mujer) & 1,66 & $0,94-2,91$ & 0,08 \\
Comorbilidad & 2,21 & $1,33-3,68$ & 0,002 \\
Índice de Garcés & 2,63 & $2,22-3,11$ & $<0,001$ \\
Superficie corporal quemada & 1,47 & $1,36-1,6$ & $<0,001$ \\
Injuria inhalatoria & 2,26 & $1,39-3,68$ & 0,001 \\
Adulto mayor & 2,66 & $1,63-4,32$ & $<0,001$ \\
Quemadura autoinfligida o "bonzo" & 2,69 & $1,41-5,15$ & 0,003 \\
Quemaduras de espesor total & 1,97 & $1,19-3,24$ & 0,008 \\
\hline
\end{tabular}

Test estadístico utilizado: Regresión multivariante de Cox.

duras profundas e injuria inhalatoria (Tabla 5). Las curvas de supervivencia estimada de Kaplan-Meier se muestran en la Figura 3 y la prueba de rango logarítmico muestra una diferencia estadística entre ambas curvas (menor curva de supervivencia en pacientes no trasladados, $\mathrm{p}<0,001)$.

La dosis letal 50 (LD50) para los pacientes no trasladados fue del $28 \%$ de la superficie corporal total quemada frente al $56 \%$ de la superficie corporal total quemada de los pacientes trasladados.

\section{Discusión}

El surgimiento de las unidades especializadas es la mejor manera de brindar atención al paciente Gran Quemado. Esto se refleja en un aumento

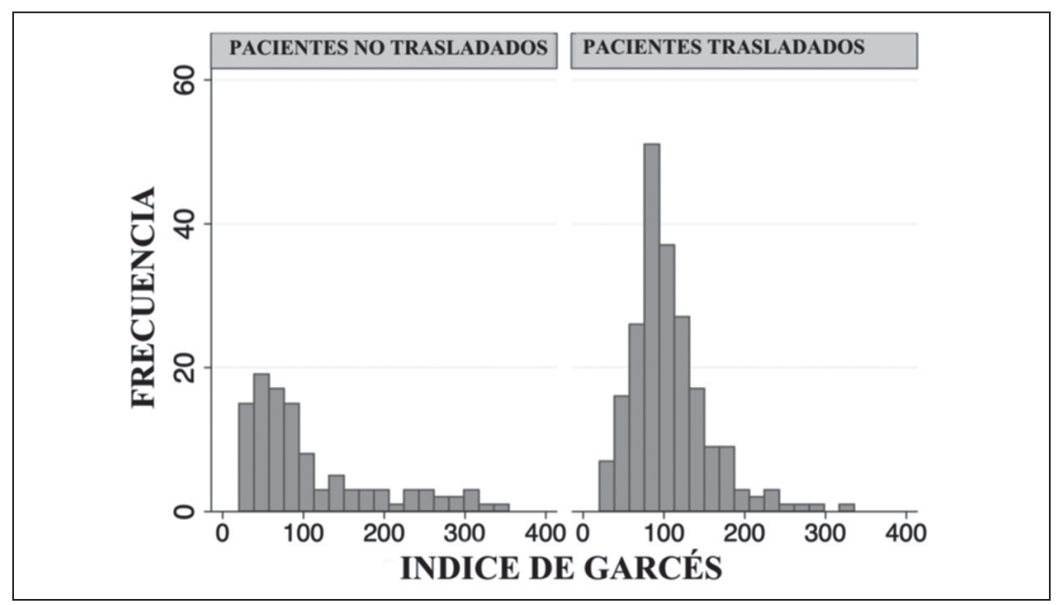

Figura 3. Índice Garcés por status de traslado. Pacientes trasladados tienen un índice de gravedad más alto. 


\section{ARTÍ́CULO ORIGINAL}

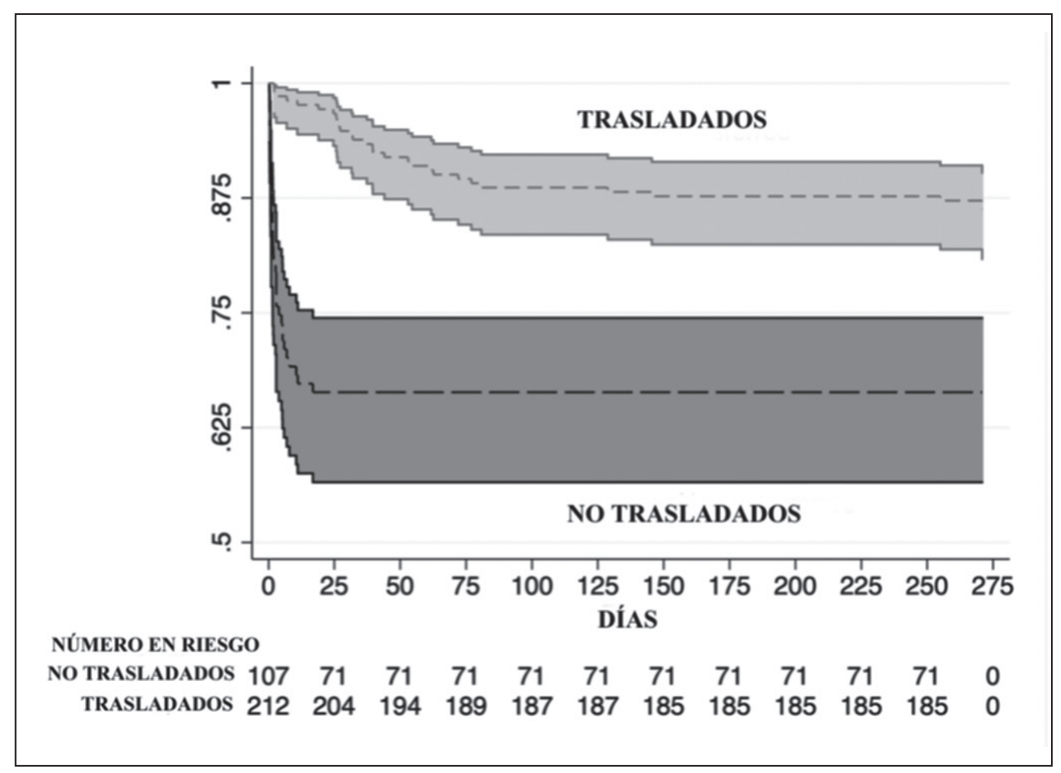

Figura 4. Curvas de supervivencia estimada de Kaplan-Meier por status de traslado. La prueba de rango logarítmico muestra una diferencia estadística significativa entre ambas curvas $(p<0,001)$ observacional descriptivo, presentado como comunicación en el Congreso Nacional de Quemados de Chile del año 2018, de todos los casos subidos al Registro Nacional de Quemados de julio a diciembre de 2017, en donde comparamos los casos que requirieron la compra de camas en el sistema de salud privado por falta de camas en nuestro centro de referencia, entre el 2016 y el 2017. Identificamos una reducción de un $42 \%$ en el número de pacientes que salieron al sistema de salud privado, y en un $25 \%$ el total de días cama. Un ahorro total de 1.745 millones de pesos chilenos, lo que significó una reducción de costos del $29 \%$ en la compra de servicios.

El Registro Nacional de Quemados incluye en su universo a aquellos pacientes que, habiendo tenido los criterios de derivación, no llegan a un centro de quemados. En ese contexto, pudimos analizar la situación del paciente quemado que no logra llegar al centro de referencia. Este grupo tiene una LD50 más baja y una mortalidad más alta, haciendo del traslado un factor protector.

Entre los factores que podrían resultar en un traslado fallido se encuentra la presencia de injuria inhalatoria, descrita hasta en un tercio de los casos ${ }^{7}$. Esta condición es uno de nuestros criterios de derivación, por lo que parece contradictorio que un paciente con esta condición sea más propenso a un traslado fallido. Creemos que el número limitado de ventiladores mecánicos y la alta ocupación de camas en nuestra unidad de pacientes críticos hacen que sea difícil, en ciertos periodos de alta demanda, conseguir un traslado precoz para ellos.

En algunos casos, donde el paciente presenta una quemadura cutánea de baja extensión, siendo el principal problema la injuria inhalatoria, nos contactamos con los médicos del hospital base para realizar el manejo del paciente en su unidad crítica y descongestionar la nuestra. La retroalimentación sobre la evolución de las quemaduras se puede realizar a través de la misma plataforma por telemedicina, la que ha demostrado buenos resultados en experiencias internacionales ${ }^{8}$.

La menor tasa de aseo quirúrgico en el hospital base en este grupo puede ser un aspecto a intervenir. Se ha visto una menor frecuencia de cirugías en el manejo de quemados en hospitales que no son centro de referencia ${ }^{5}$. En nuestro país los cirujanos generales no siempre están entrenados en el manejo quirúrgico del paciente quemado y en la necesidad de un aseo quirúrgico diagnóstico temprano.

Creemos que este grupo de pacientes no trasladados constituyen un grupo de riesgo sobre el que implementar medidas de salud pública, por ejemplo, 
aumentar el número de camas de UCI y ventiladores en nuestro centro de quemados.

\section{Conclusión}

La implementación de una plataforma de registro en línea puede tomarse como modelo en otros países en vías de desarrollo para optimizar el flujo de estos pacientes, ahorrar recursos y contar con nuevas fuentes de información epidemiológica.

\section{Responsabilidades éticas}

Protección de personas y animales. Los autores declaran que para esta investigación no se han realizado experimentos en seres humanos ni en animales.

Confidencialidad de los datos. Los autores declaran que en este artículo no aparecen datos de pacientes.

Conflictos de interés: no hay.

\section{Bibliografía}

1. Ministerio de Salud de Chile. Guías Clínicas AUGE Gran Quemado. 2016 [Consultado el 7 Febrero 2020] Disponible en: http://www.bibliotecaminsal.cl/wp/wpcontent/uploads/2016/04/GPC-GRANQUEMADO-FINAL-18-MARZO-2016 DIAGRAMADA.pdf.

2. Albornoz CR, Villegas J, Peña V, Whittle $\mathrm{S}$. Epidemiología del paciente gran quemado adulto en Chile: experiencia del Servicio de Quemados del Hospital de la Asistencia Pública de Santiago. Rev Med Chile 2013;141:181-6.

3. Registro Nacional de Quemados
[Internet]. 2021. Disponible en: http:// quemados.minsal.cl/ [Consultado el 7 Febrero 2020].

4. Miller SF, Bessey P, Lentz CW, Jeng JC, Schurr M, Browning S. National Burn Repository 2007 Report: A Synopsis of the 2007 Call for Data. J Burn Care Res. 2008;29:862-70.

5. Prindeze NJ, Marano MA, Shupp JW, Johnson LS. Cross-Sectional Assessment of Burn Center and Nonburn Center Patient Care: A Multi-Database Comparison. J Burn Care Res. 2020 Disponible en: https://doi.org/10.1093/ jbcr/iraa006.

6. Al-Mousawi AM, Mecott-Rivera GA,
Jeschke MG, Herndon DN. Burn Teams and Burn Centers: The Importance of a Comprehensive Team Approach to Burn Care. Clin Plast Surg. 2009;36:547-54.

7. Chung KK, Rhie RY, Lundy JB, Cartotto R, Henderson E, Pressman MA, et al. A Survey of Mechanical Ventilator Practices Across Burn Centers in North America. J Burn Care Res. 2016;37: e131-9.

8. Monte A, López B, Aguilera J,Serracanta J, Collado JM, Moreno C, et al. Implementation and evaluation of telemedicine in burn care: Study of clinical safety and technical feasibility in a single burn center. Burns 2020;46:166873. 